\title{
REVIEW ARTICLE \\ Dose-specific efficacy of Haemophilus influenzae type b conjugate vaccines: a systematic review and meta-analysis of controlled clinical trials
}

\author{
U. K. GRIFFITHS ${ }^{1 *}$, A. CLARK ${ }^{2}$, B. GESSNER ${ }^{3}$, A. MINERS ${ }^{2}$, C. SANDERSON ${ }^{2}$, \\ E. R. SEDYANINGSIH ${ }^{4}$ AND K. E. MULHOLLAND ${ }^{5}$ \\ ${ }^{1}$ Department of Global Health and Development, London School of Hygiene and Tropical Medicine, London, UK \\ ${ }^{2}$ Department of Health Services Research and Policy, London School of Hygiene and Tropical Medicine, UK \\ ${ }^{3}$ Agence de Medecine Preventive, Paris, France \\ ${ }^{4}$ Ministry of Health, Indonesia \\ ${ }^{5}$ Department of Infectious Diseases and Epidemiology, London School of Hygiene and Tropical Medicine, UK
}

Received 7 December 2011; Final revision 16 April 2012; Accepted 16 April 2012;

first published online 14 May 2012

\section{SUMMARY}

Global coverage of infant Haemophilus influenzae type b (Hib) vaccination has increased considerably during the past decade, partly due to GAVI Alliance donations of the vaccine to low-income countries. In settings where large numbers of children receive only one or two vaccine doses rather than the recommended three doses, dose-specific efficacy estimates are needed to predict impact. The objective of this meta-analysis is to determine Hib vaccine efficacy against different clinical outcomes after receiving one, two or three doses of vaccine. Studies were eligible for inclusion if a prospective, controlled design had been used to evaluate commercially available Hib conjugate vaccines. Eight studies were included. Pooled vaccine efficacies against invasive Hib disease after one, two or three doses of vaccine were $59 \%, 92 \%$ and $93 \%$, respectively. The meta-analysis provides robust estimates for use in decision-analytical models designed to predict the impact of Hib vaccine.

Key words: Efficacy, Haemophilus influenzae, meta-analysis, systematic review, vaccine.

\section{INTRODUCTION}

Haemophilus influenzae type b (Hib) is an encapsulated, Gram-negative coccobacillus that can cause childhood meningitis, pneumonia and a number of rarer forms of disease, such as epiglottitis, septicaemia and cellulitis [1]. Hib conjugate vaccines have gradually been introduced into more and more routine programmes since the early 1990s. While 89 countries used the vaccine in 2004, the number had increased to

\footnotetext{
* Author for correspondence: Dr U. K. Griffiths, Department of Global Health and Development, London School of Hygiene and Tropical Medicine, 15-17 Tavistock Place, London WC1H 9SH, UK.

(Email: ulla.griffiths@1shtm.ac.uk)
}

172 countries by 2011 [2]. In low- and lower-middleincome countries, introduction of the vaccine has to a large extent been facilitated by support from the GAVI Alliance [3].

The first Hib vaccine was produced in the early 1970s and composed of purified Hib capsular polysaccharide. However, since this vaccine was only effective in children aged $>2$ years it was not widely used [4]. More immunogenic vaccines were developed in the late 1980 s by conjugating capsular polysaccharides to protein carriers. Four different Hib conjugate vaccines have been developed, diverging in the carrier protein, the method of conjugation, and in the structure and lengths of the polysaccharide

The online version of this article is published within an Open Access environment subject to the conditions of the Creative Commons Attribution-NonCommercial-ShareAlike licence $<$ http://creativecommons.org/licenses/by-nc-sa/2.5/ $>$. The written permission of Cambridge University Press must be obtained for commercial re-use. 
polyribosolribitol phosphate (PRP) element [4]. The four vaccine types are known as PRP-D, PRP-OMP, HbOC and PRP-T. The PRP-D vaccine was licensed following a randomized controlled trial (RCT) in Finnish children [5], but when it subsequently proved ineffective in preventing Hib disease in Alaskan natives [6], production was stopped. Hence, today only the other three vaccines are in use. While the World Health Organization does not differentiate between the three vaccines [7], they are not identical; for example, PRP-OMP induces a substantial antibody response after a single dose while PRP-T and HbOC require two doses [8].

Vaccine efficacy is measured in RCTs with the aim of determining whether infection is reduced under ideal conditions designed to maximize disease detection [9]. The objective of this meta-analysis is to determine dose-specific efficacy of commercially available Hib vaccines against different clinical outcomes. These estimates are important parameter values when modelling the impact and cost-effectiveness of Hib vaccine [10]. In simple models, the vaccinepreventable disease burden is estimated as:

$$
\underset{\text { incidence }}{\text { disease }} \times \underset{\text { coverage }(3 \text { doses })}{\text { vaccine }} \times \underset{\text { efficacy }(3 \text { doses })}{\text { vaccine }} .
$$

While such estimates will clearly exclude the potential indirect 'herd immunity' benefits for unvaccinated children, they will also underestimate the direct impact attributed to partially vaccinated children. In low- and lower-middle-income settings with weak health systems, many children receive only one or two vaccine doses rather than the recommended three. Since vaccine efficacy will be lower in these partially vaccinated children, dose-specific estimates of vaccine efficacy are required to improve estimates of expected vaccine impact. If possible, other factors, such as late vaccination, should also be incorporated into estimates of the direct effect of a vaccination programme [11]. Moreover, with more new vaccines being introduced, such as pneumococcal and rotavirus vaccines, the routine vaccination schedule is being questioned with regard to the appropriate age and timing of vaccination [12]. Pooled dose-specific efficacy estimates from all RCTs are a crucial piece of information needed to guide these considerations.

One other meta-analysis of Hib vaccine RCTs was identified before the present review was started. Obonyo \& Lau searched the literature until 2005 and included eight studies, two of which were of the PRP-D vaccine [13]. The pooled efficacy estimate against invasive Hib disease was found to be $84 \%$ [95\% confidence interval (CI) 70-99]. As is common in the reporting of vaccine trial results, few dose-specific values were presented.

Vaccine effectiveness studies are designed to measure protectiveness under field conditions. These can either be a prospective cohort study, a casecontrol study, or data can be accumulated retrospectively from routine surveillance. For establishing Hib vaccine effectiveness the case-control method, where a group of cases with the target outcome, most frequently Hib meningitis, is contrasted with a group of controls who did not develop the target outcome, is the most commonly used method [14]. An important challenge of case-control studies is, however, to produce convincing evidence that the vaccinated and unvaccinated populations are sufficiently alike in all relevant characteristics other than vaccination to allow a reasonable conclusion that the differences between groups are attributable to vaccine efficacy. A meta-analysis of Hib vaccine effectiveness studies was published by O'Loughlin et al. in 2010 [14]. Twenty-five studies, of which 14 were case-control studies, were identified. Three of the studies reported vaccine effectiveness against invasive Hib disease after three doses and the pooled estimate was $95 \%(95 \%$ CI 82-99). One of the conclusions from this review was that the effectiveness of Hib vaccine has been well documented and the need for more case-control studies is minimal [14].

\section{Search strategy and study selection}

Recommendations of the Preferred Reporting Items for Systematic Reviews and Meta-Analyses (PRISMA) statement were used [15]. Studies were identified from the Cochrane Controlled Trials Register (CCTR) using the search term 'haemophilus vaccine' in March 2011. The CCTR is a bibliographical database of controlled trials systematically identified from Medline and EMBASE by contributors of the Cochrane Collaboration [16]. Reference lists in identified papers were checked and experts in the field were contacted to confirm that no studies had been missed.

Studies were eligible for inclusion if they compared a commercially available Hib vaccine with placebo, if clinical endpoints were reported, and if participants had been allocated prospectively using random or quasi-random allocation. To maximize the amount of data available, quality measures were not used as exclusion criteria. There were no language restrictions. 


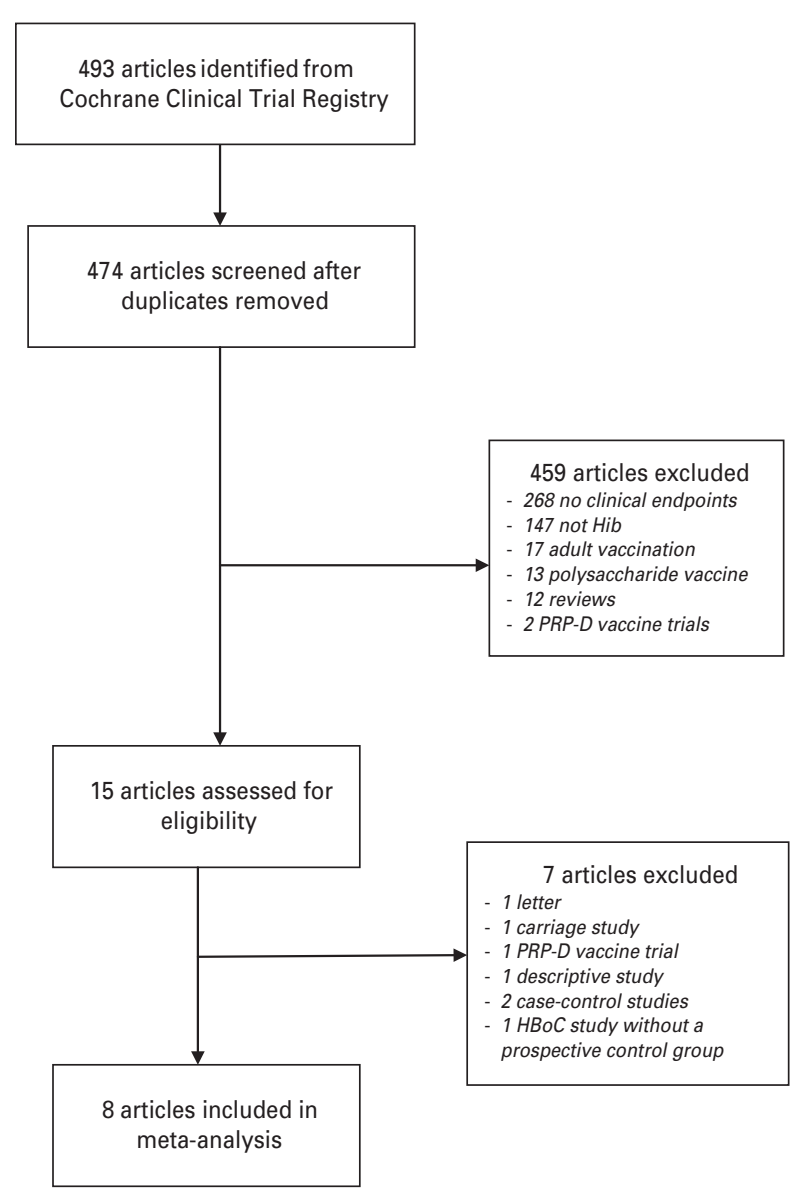

Fig. 1. Study selection.

Data extraction and quality assessment were performed by two authors independently (U.K.G. and A.C.). Extracted data included setting, type of Hib vaccine, schedule, dosage, duration, number of Hib disease cases according to study group, and total numbers of children in both groups. Cochrane's Risk of Bias tool was used to assess the methodological quality of the trials [17].

\section{Outcome measures}

In most epidemiological studies of Hib disease, focus is solely placed on invasive disease, defined as the isolation of Hib from a sterile site such as the bloodstream, synovial fluid or cerebrospinal fluid. Noninvasive disease occurs when Hib has been isolated from a non-sterile site, such as an external ear or throat swab. All severe types of Hib diseases, such as pneumonia, meningitis, epiglottitis and sepsis, are invasive. However, the aetiology of invasive pneumonia is intrinsically difficult to determine [18]. The only method which would reliably determine the aetiology is culture of lung aspirate, but this procedure is invasive and not ethically feasible in all RCTs. Moreover, selection bias will occur if antibiotics are commonly used before seeking healthcare as this will markedly reduce the sensitivity of the lung aspirate test [19]. Blood culture is only useful when pneumonia is associated with bacteraemia, which is only in a fraction of cases. The yield from blood culture in patients with Hib pneumonia has ranged from $10 \%$ to $30 \%[20,21]$. Hence, since there are currently no validated and specific methods for confirming the pathogen-specific bacterial aetiology of pneumonia, several non-specific pneumonia endpoints have been used in Hib vaccine RCTs.

The following outcomes were included in the metaanalysis: (i) confirmed invasive Hib disease, (ii) confirmed Hib meningitis, (iii) confirmed Hib pneumonia, (iv) clinical meningitis, (v) radiologically (or chest X-ray) confirmed pneumonia, (vi) hospitalized pneumonia and (vii) clinical pneumonia. In addition, efficacy estimates were calculated according to the number of doses of Hib vaccine received as either (i) only one dose, (ii) only two doses or (iii) three doses.

\section{Statistical analysis}

All statistical analyses were performed with Stata version 11 (StataCorp., USA). Trial results were expressed as percent vaccine efficacy, defined as $1-$ relative risk. Estimates were pooled using a randomeffects model applying the DerSimonian \& Laird approach [22]. Heterogeneity was assessed using the $\chi^{2}$ test with a $P$ value of $<0 \cdot 10$ indicating statistical significance and by the $I^{2}$ statistic, which measures the percentage of variation attributable to heterogeneity. A value of $0 \%$ indicates no observed heterogeneity and larger values show increasing heterogeneity [23].

\section{RESULTS}

\section{Characteristics of eligible trials}

The search strategy yielded 493 references (Fig. 1). After removing duplicates, 474 titles and abstracts were screened and 459 papers excluded. The most common reason for exclusion at this stage was that the study reported only safety and immunogenicity of the vaccine with no clinical endpoints. Fifteen papers were retrieved for full-text review and seven of these were excluded. One of the excluded studies was a 


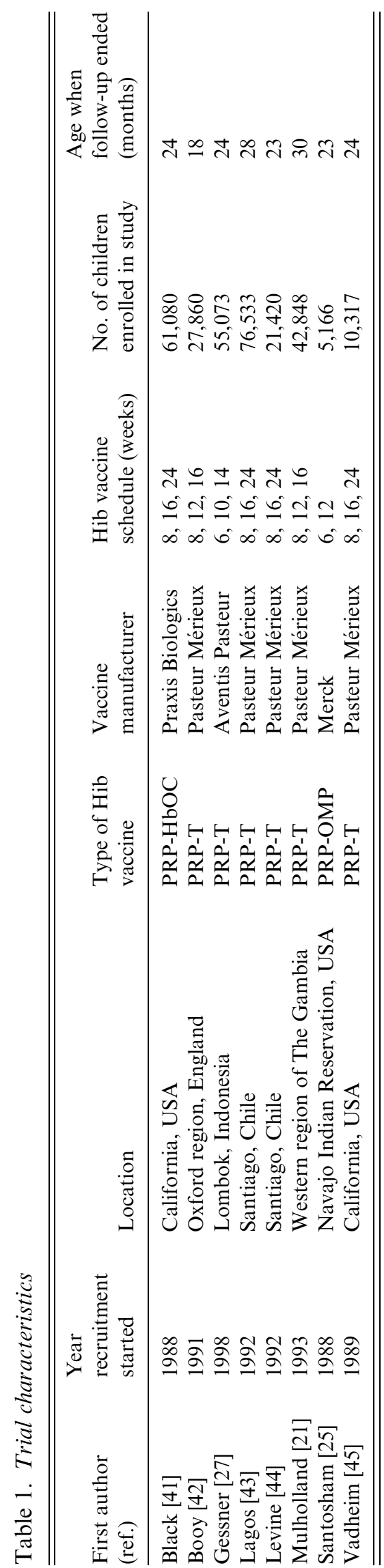

large, Finnish prospective study where children born on even-numbered days received $\mathrm{HbOC}$ vaccine and those born on uneven-numbered days received PRP-D vaccine [24]. Vaccine efficacy was estimated by comparing the number of Hib disease cases during the study period with the number expected based on historical routine surveillance data. The study was excluded because the comparison group was historical, not prospective.

Six of the eight included papers evaluated PRP-T, one HbOC and one PRP-OMP vaccines (see Table 1). The studies by Lagos and Levine analysed the same trial participants. Three of the studies were from the USA and the remaining from England, Chile, The Gambia and Indonesia. One of the US studies was conducted in a Navajo Indian reservation, targeting a group with one of the highest incidences of Hib disease in the world [25]. In this trial only two doses of Hib vaccine were administered, while three doses were evaluated in the seven remaining studies. The study by Vadheim ended prematurely due to inclusion of the $\mathrm{HbOC}$ vaccine in the US routine vaccination schedule while the study was ongoing, so no vaccine efficacy estimates were reported because the sample sizes were too small to achieve sufficient power. In all the studies, follow-up stopped after a specified time period. The study by Booy had the shortest follow-up time and the study by Mulholland the longest, with the oldest children being aged 18 and 30 months, respectively, when the studies ended (Table 1).

Outcome measures used in the trials are summarized in Table 2. The outcome measure used by most of the studies was invasive Hib disease. While all types of Hib diseases are included within the invasive disease group, meningitis is likely to be the most frequent type of syndrome detected. In the study by Gessner, the only outcome measure that included aetiological confirmation was Hib meningitis. The studies by Booy, Levine and Vadheim did not report vaccine efficacy for less than three vaccine doses.

Clinical, non-specific pneumonia outcomes were included in the RCTs from Chile, The Gambia and Indonesia. Since pneumonia disease incidence is considerably less in high-income than low-income countries, non-specific pneumonia endpoints were not relevant to include in the RCTs from England and the USA. However, in low-income countries prevention of pneumonia may be the primary argument for introducing Hib vaccine, so it was crucial for the studies in these countries to measure efficacy against pneumonia. 


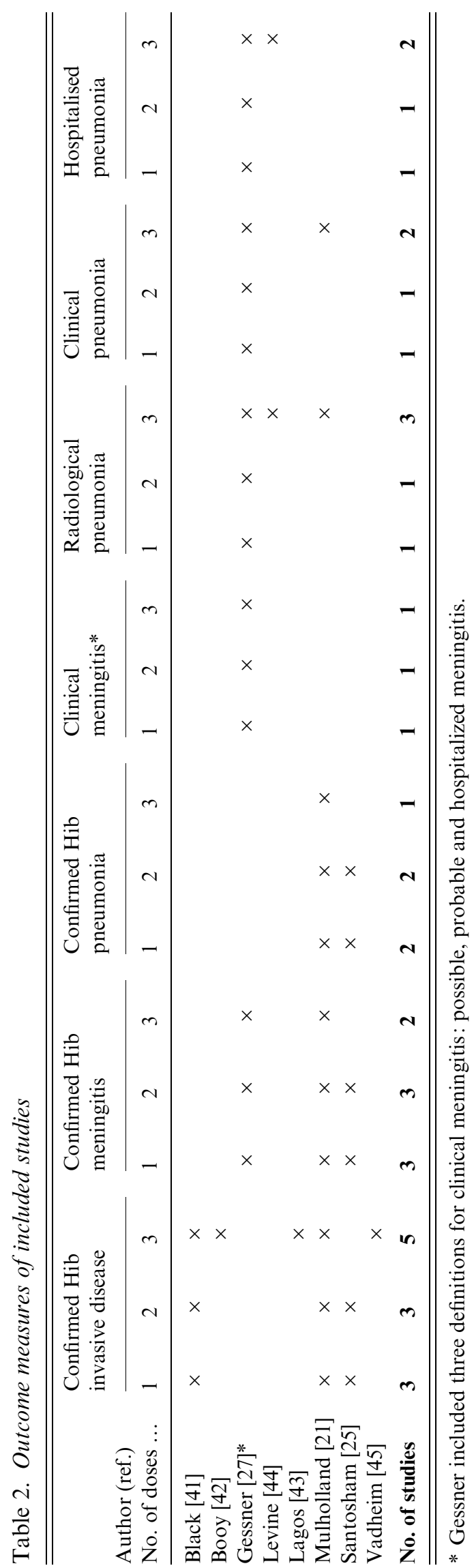

\section{Methodological quality of trials and risk of bias}

The domains specified in Cochrane's Risk of Bias tool are summarized in Table 3. Risks of bias due to randomization, sequence generation and allocation concealment were relatively low in all the studies. Even though two studies were not randomized, it seems unlikely that the results would be biased for this reason. However, four of the studies were not blinded and this leads to risk of bias. In the three studies where confirmed Hib disease was the only endpoint, the risk of bias would most likely be low because the microbiologists undertaking the tests for Hib were not aware of the child's vaccination status. However, in the Levine study, radiological and hospitalized pneumonia were the chosen endpoints and assessments of these were made from hospital records of children with known vaccination status, leading to moderate risk of bias. With regard to inclusion of all randomized participants, all the studies suffer from high risk of bias due to the inherent difficulties of diagnosing Hib. Even in the studies with enhanced surveillance, it is possible that not all Hib cases were detected. The risk of detection bias is especially large in the three studies with non-specific (or all-cause) pneumonia as an outcome.

An additional risk of bias in the studies using non-specific pneumonia outcomes is that estimated Hib vaccine efficacy will vary with the relative proportion of Hib pneumonia occurring during the study period. Since many of the other pathogens that cause pneumonia are known to be seasonal, such as for instance respiratory synical virus (RSV) or influenza, this could be a problem. Hib vaccine efficacy against clinical pneumonia would for instance be less in a year with a RSV pneumonia outbreak than in a year with low RSV incidence [26]. Since all the trials lasted for more than one year, seasonal fluctuations might have been evened out to some extent, but the risk of this bias is apparent. This is also an important limitation to bear in mind when generalizing the efficacy values to other settings where the relative proportion of Hib pneumonia may be different.

The risk of publication bias is considered low for vaccine trials. Since these are large trials involving thousands of children and lasting several years, there is strong pressure on investigators and vaccine manufacturers to publish the results. It is thus highly unlikely that any Hib vaccine RCTs have taken place without being published. 
Table 3. Assessment of risk of bias in the included trials

\begin{tabular}{|c|c|c|c|c|c|}
\hline $\begin{array}{l}\text { First author } \\
\text { (ref.) }\end{array}$ & $\begin{array}{l}\text { Unit of } \\
\text { randomization }\end{array}$ & Sequence generation & Blinding & Allocation concealment & Outcome reporting \\
\hline Black [41] & Individual & $\begin{array}{l}\text { Placebo group was } \\
\text { refusers and children } \\
\text { born on the first } \\
5 \text { days of a month }\end{array}$ & $\begin{array}{l}\text { No, but microbiologists } \\
\text { performing tests for } \mathrm{Hib} \\
\text { were unaware of } \\
\text { vaccination status }\end{array}$ & n.a. & $\begin{array}{l}\text { Enhanced surveillance to detect } \\
\text { all Hib patients treated in } \\
\text { the study area }\end{array}$ \\
\hline Booy [42] & Cluster & $\begin{array}{l}\text { Hib vaccine districts } \\
\text { determined from } \\
\text { availability of } \\
\text { computer systems }\end{array}$ & $\begin{array}{l}\text { No, but microbiologists } \\
\text { performing tests for } \mathrm{Hib} \\
\text { were unaware of } \\
\text { vaccination status }\end{array}$ & n.a. & $\begin{array}{l}\text { Enhanced surveillance to detect } \\
\text { all Hib patients treated in } \\
\text { the study area }\end{array}$ \\
\hline Gessner [27] & Cluster & Random & Double blinding & $\begin{array}{l}\text { Vaccine vials identical } \\
\text { except four colour codes; } \\
\text { two for Hib and two for placebo. } \\
\text { Code in a locked vault }\end{array}$ & $\begin{array}{l}\text { Education for diagnosis and } \\
\text { referral. Young women in every } \\
\text { village to identify children with } \\
\text { pneumonia and get them to hospital. } \\
\text { Families reimbursed costs }\end{array}$ \\
\hline Lagos [43] & Cluster & Random & $\begin{array}{l}\text { No, but microbiologists } \\
\text { performing tests for } \mathrm{Hib} \\
\text { were unaware of } \\
\text { vaccination status }\end{array}$ & n.a. & $\begin{array}{l}\text { Active surveillance at } 11 \text { hospitals } \\
\text { in Santiago. Bacteriology laboratory } \\
\text { reports reviewed weekly }\end{array}$ \\
\hline Levine [44] & Cluster & Random & No & n.a. & $\begin{array}{l}\text { Retrospective review of five } \\
\text { ICD-9 pneumonia } \\
\text { discharge diagnoses }\end{array}$ \\
\hline Mulholland [21] & Individual & Random & Double blinding & $\begin{array}{l}\text { Five vaccine vial codes used } \\
\text { for Hib and five others for } \\
\text { placebo. Only safety monitoring } \\
\text { group knew the code }\end{array}$ & $\begin{array}{l}\text { Study children presenting to } \\
\text { health centres due to any illness } \\
\text { referred to study physician }\end{array}$ \\
\hline Santosham [25] & Cluster & Random & Double blinding & $\begin{array}{l}\text { Vaccine and placebo vials had } \\
\text { similar appearances. Code not } \\
\text { known until end of study }\end{array}$ & $\begin{array}{l}\text { Active and passive } \\
\text { surveillance throughout } \\
\text { study area }\end{array}$ \\
\hline Vadheim [45] & Individual & Random & Double blinding & No details given on method used & $\begin{array}{l}\text { Active and passive surveillance } \\
\text { throughout study area }\end{array}$ \\
\hline
\end{tabular}

n.a., Not available. 


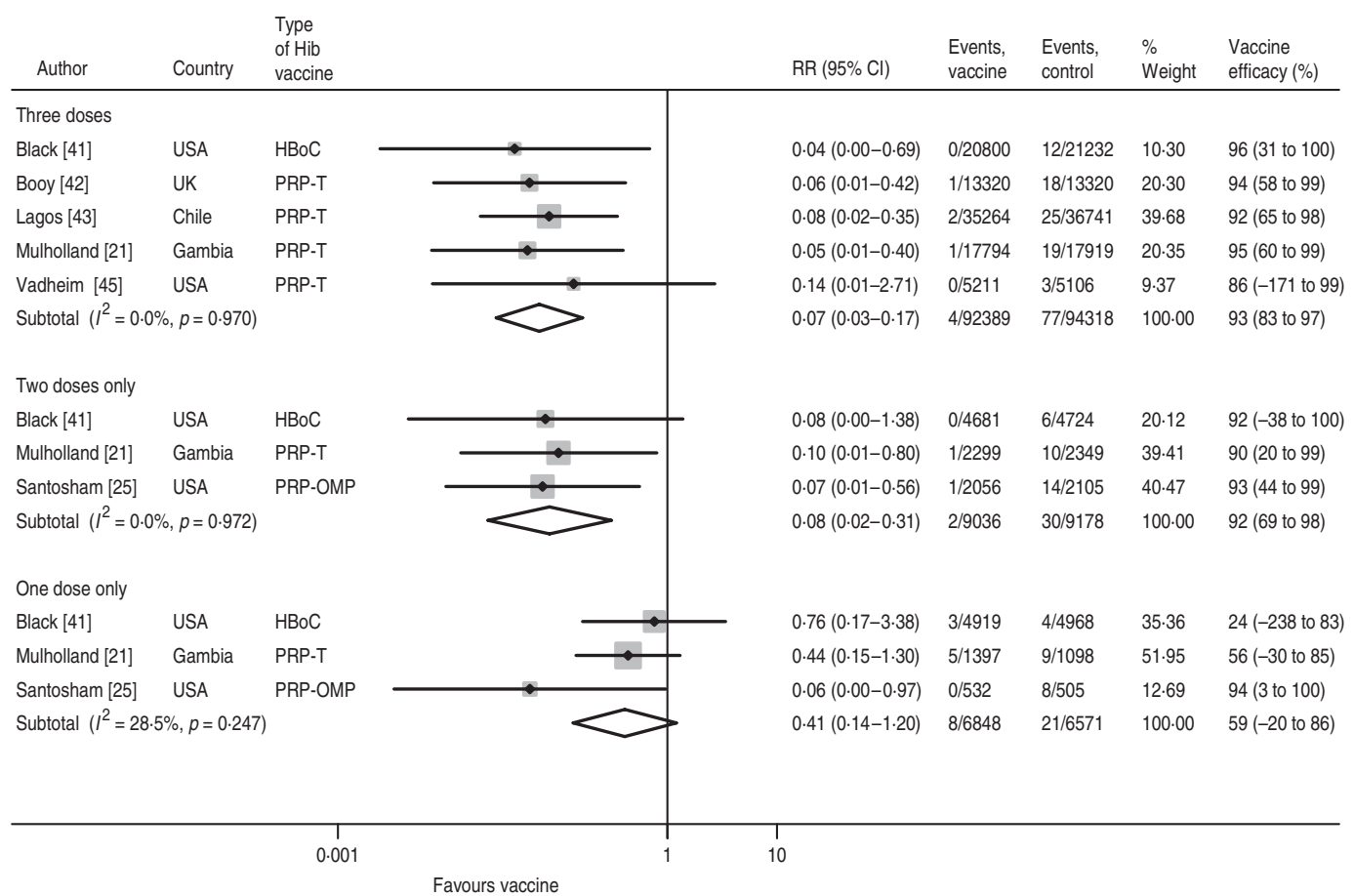

Fig. 2. Hib vaccine efficacy against confirmed invasive Hib disease.

\section{Vaccine efficacy}

The pooled efficacy estimates against confirmed, invasive Hib disease following three, two and one dose are $93 \%$ (95\% CI 83-97), $92 \%$ (95\% CI 69-98) and $59 \%$ (95\% CI -20 to 86$)$, respectively (Fig. 2). There is no heterogeneity in the studies for three and two doses and only low heterogeneity for one dose $\left(I^{2}=28.5 \%\right)$, which is attributable to the Santosham study of the PRP-OMP vaccine reporting considerably higher efficacy than the two other studies. The pooled one-dose estimate of the trials using the PRP-T and the HbOC vaccines, respectively, is $47 \%(95 \%$ CI -28 to 70$)$ with no heterogeneity $\left(I^{2}=0.0 \%\right)$. The children in the vaccine groups who experienced Hib disease after one or two doses remained incompletely immunized during the course of the studies. Hence, the cases did not occur during the inter-vaccination intervals.

For confirmed Hib meningitis there is no heterogeneity in any of the dose-specific estimates, but due to the relatively small sample sizes, the confidence intervals are wide for one and two doses (Fig. 3). Moreover, since the Santosham trial only estimated one- and two-dose efficacy, the pooled value for three doses $(88 \%, 95 \%$ CI $46-97)$ is less than for two doses $(92 \%, 95 \%$ CI 37-99). The pooled one-dose estimate is $62 \%(95 \% \mathrm{CI}-29$ to 89$)$.
Data on confirmed Hib pneumonia were only available from the Mulholland and Santosham studies, and Santosham did not identify any cases following two doses. The Mulholland midpoint efficacy estimates of both two and three doses were $91 \%$ ( $95 \%$ CI -66 to 99 ) and the pooled estimate between Mulholland and Santosham following one dose was $67 \%$ (95\% CI -44 to 93 ).

Vaccine efficacy against radiological pneumonia with three doses was $22 \%$ in both the Levine and Mulholland studies, but the result was negative in the study by Gessner, causing large heterogeneity $\left(I^{2}=68.9 \%\right)$ (Fig. 4). Hence, the pooled estimate has limited validity. The pooled estimate without the Gessner study was $22 \%$ (95\% CI 6-35) with no heterogeneity $\left(I^{2}=0 \cdot 0 \%\right)$.

Hib vaccine efficacy against hospitalized pneumonia following three doses of vaccine was reported by Gessner and Levine (Fig. 4). There is, however, also large heterogeneity between these two studies and the pooled estimate is not meaningful. While Levine found a vaccine efficacy of $26 \%(95 \%$ CI $7-41)$, Gessner only estimated it as $2 \%(95 \% \mathrm{CI}-8$ to 10$)$.

Clinical pneumonia efficacy after three doses was reported by Gessner and Mulholland and found to be $4 \%(95 \%$ CI 1-7) in both studies (Fig. 4). However, due to the larger number of cases detected 


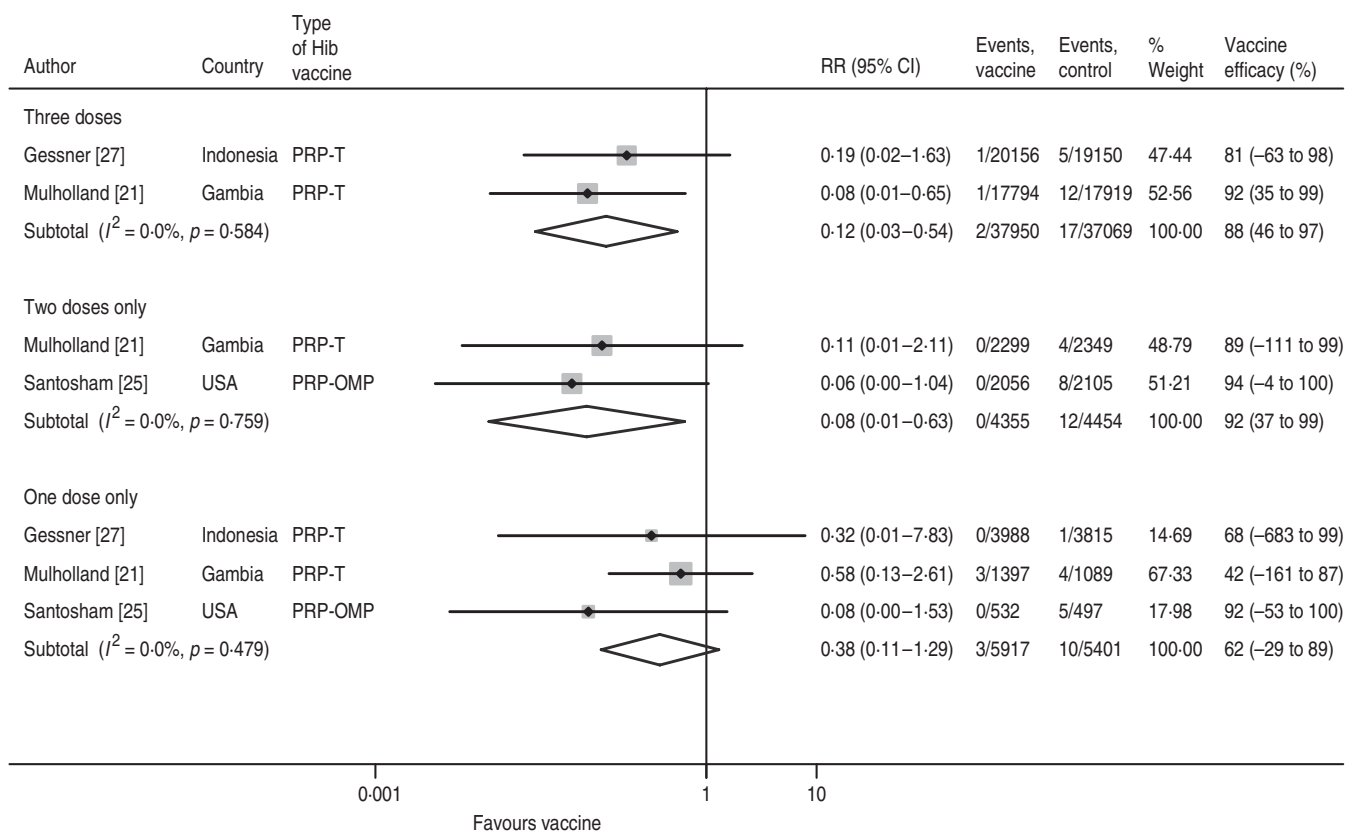

Fig. 3. Hib vaccine efficacy against confirmed Hib meningitis.

\begin{tabular}{|c|c|c|c|c|c|c|}
\hline Author & Country & RR $(95 \% \mathrm{Cl})$ & $\begin{array}{l}\text { Events, } \\
\text { vaccine }\end{array}$ & $\begin{array}{l}\text { Events, } \\
\text { control }\end{array}$ & $\begin{array}{l}\% \\
\text { Weight }\end{array}$ & $\begin{array}{l}\text { Vaccine } \\
\text { efficacy (\%) }\end{array}$ \\
\hline \multicolumn{7}{|l|}{ Hospitalised } \\
\hline Gessner [27] & Indonesia & $0.98(0.90-1.08)$ & $926 / 19934$ & $893 / 18916$ & $57 \cdot 12$ & $2(-8$ to 10$)$ \\
\hline Levine [44] & Chile & $0.74(0.59-0.93)$ & $131 / 11146$ & $163 / 10274$ & 42.88 & 26 (7 to 41$)$ \\
\hline Subtotal $\left(I^{2}=80\right.$ & $p=0.023)$ & $0.87(0.66-1.15)$ & $1057 / 31080$ & $1056 / 29190$ & $100 \cdot 00$ & $13(-15$ to 34$)$ \\
\hline \multicolumn{7}{|l|}{ Radiological } \\
\hline Gessner [27] & Indonesia & $1.10(0.91-1.33)$ & $221 / 20102$ & $191 / 19104$ & 37.83 & $-10(-33$ to 9$)$ \\
\hline Levine [44] & Chile & $0.78(0.57-1.07)$ & $72 / 11146$ & $85 / 10274$ & $27 \cdot 41$ & $22(-7$ to 43$)$ \\
\hline Mulholland [21] & Gambia & $0.78(0.62-0.98)$ & $132 / 17794$ & $170 / 17919$ & 34.75 & 22 (2 to 38$)$ \\
\hline Subtotal $\left(I^{2}=68\right.$ & $p=0.042)$ & $0.89(0.70-1.14)$ & $425 / 49042$ & $446 / 47297$ & $100 \cdot 00$ & $11(-14$ to 30$)$ \\
\hline \multicolumn{7}{|l|}{ Clinical } \\
\hline Gessner [27] & Indonesia & $0.96(0.93-1.00)$ & $4957 / 18762$ & $4870 / 17748$ & $92 \cdot 26$ & $4(0$ to 7$)$ \\
\hline Mulholland [21] & Gambia & $0.96(0.85-1.08)$ & $526 / 17794$ & $570 / 18489$ & 7.74 & $4(-8$ to 15$)$ \\
\hline \multicolumn{2}{|c|}{ Subtotal $\left(I^{2}=0.0 \%, p=0.946\right)$} & $0.96(0.93-0.99)$ & $5483 / 36556$ & $5440 / 36237$ & $100 \cdot 00$ & $4(1$ to 7$)$ \\
\hline & 0.01 & $\begin{array}{c}1 \\
10\end{array}$ & & & & \\
\hline
\end{tabular}

Fig. 4. Hib vaccine efficacy against non-specific pneumonia outcome measures.

in Indonesia compared to The Gambia, the weight of the Gessner study in the pooled estimate is as high as $92 \cdot 3 \%$.

Clinical meningitis was only included in the study by Gessner and three different types were included; probable, possible and hospitalized meningitis (Table 4). There were consistently less cases in the vaccinated compared to the placebo group and two of the efficacy estimates were significant at the $95 \%$ level. This indicated that, in the Indonesian environment, many cases of Hib meningitis were reaching a health facility, but either did not have a lumbar puncture or lumbar puncture was done and found to have the microscopic appearance of meningitis, but for reasons of specimen handling or microbiological procedures, Hib was not cultured. 
Table 4. Vaccine efficacy against clinical meningitis in Lombok, Indonesia

\begin{tabular}{|c|c|c|c|c|c|c|c|}
\hline \multirow[b]{2}{*}{ Outcome measure } & \multirow{2}{*}{$\begin{array}{l}\text { No. of Hib } \\
\text { vaccine doses }\end{array}$} & \multicolumn{2}{|c|}{ Cases/total children } & \multirow{2}{*}{$\begin{array}{l}\text { Vaccine } \\
\text { efficacy }\end{array}$} & \multicolumn{2}{|c|}{$95 \% \mathrm{CI}$} & \multirow[b]{2}{*}{$P$ value } \\
\hline & & $\mathrm{Hib}$ & Placebo & & Low & High & \\
\hline Probable bacterial meningitis & 1 & $1 / 3989$ & $6 / 3816$ & $84 \%$ & -32 & 98 & $0 \cdot 06$ \\
\hline Probable bacterial meningitis & 2 & $5 / 4006$ & $6 / 3963$ & $18 \%$ & -170 & 75 & $0 \cdot 77$ \\
\hline Probable bacterial meningitis & 3 & $9 / 20152$ & $20 / 19147$ & $57 \%$ & 6 & 81 & $0 \cdot 04^{*}$ \\
\hline Possible bacterial meningitis & 1 & $3 / 3989$ & $9 / 3819$ & $68 \%$ & -18 & 91 & $0 \cdot 09$ \\
\hline Possible bacterial meningitis & 2 & $6 / 4006$ & $6 / 3963$ & $1 \%$ & -206 & 68 & $1 \cdot 00$ \\
\hline Possible bacterial meningitis & 3 & $17 / 20152$ & $34 / 19144$ & $53 \%$ & 15 & 73 & $0 \cdot 01^{*}$ \\
\hline Hospitalized meningitis & 1 & $21 / 3993$ & $34 / 3824$ & $41 \%$ & -2 & 66 & $0 \cdot 06$ \\
\hline Hospitalized meningitis & 2 & $33 / 4010$ & $41 / 3971$ & $20 \%$ & -26 & 49 & $0 \cdot 35$ \\
\hline Hospitalized meningitis & 3 & $118 / 20144$ & $121 / 19131$ & $7 \%$ & -19 & 28 & $0 \cdot 56$ \\
\hline
\end{tabular}

CI, Confidence interval.

* Significant at the 95\% level using Fisher's exact $t$ test.

\section{DISCUSSION}

All the studies demonstrated high vaccine efficacy against confirmed invasive Hib disease following two and three doses, and there was no heterogeneity in the pooled estimate. With one dose, the pooled estimate was $59 \%(95 \% \mathrm{CI}-20$ to 86$)$ and there was only low heterogeneity. Hence, the vaccine efficacy evidence against invasive disease is convincing and robust for all dose regimens. This conclusion is similar to the findings of O'Loughlin and colleagues in their metaanalysis of observational studies [14]. The pooled vaccine effectiveness estimate against invasive $\mathrm{Hib}$ disease from three case-control studies was $95 \%$ (95\% CI 82-99) after three doses and $92 \%$ (95\% CI 81-97) following two doses. In the meta-analysis of RCTs by Obonyo \& Lau, vaccine efficacy against invasive Hib disease after three doses was only $84 \%$ (95\% CI 69-92). The reason for the higher pooled estimate in the new analysis is partly the exclusion of the PRP-D trials, but also that Obonyo \& Lau combined all doses into one analysis, so that children receiving only one dose, only two doses and all three doses were included in the same estimate.

The evidence for confirmed Hib meningitis and confirmed Hib pneumonia is less robust because few studies report on these, so the pooled estimates are based on small sample sizes, which give wide confidence intervals. As both diseases are contained within the overall group of invasive Hib disease, these efficacy estimates should be used instead of seeking to categorize according to invasive Hib disease type.

As expected, the strength of the evidence of vaccine efficacy against the three, non-specific pneumonia outcomes is less than for confirmed Hib disease. For hospitalized pneumonia the heterogeneity is too large for the efficacy estimate to be meaningful. The large heterogeneity between the Indonesian and Chilean studies may partly be due different case detection methods. Pneumonia detection in Indonesia was based on individual presentation for medical care, but supported by village health workers whose task was to identify children in the community with possible severe respiratory disease and refer them for treatment. By contrast, cases were retrospectively identified from patient records in Chile. Another possible explanation is differences between the two sites in the distribution of non-Hib pneumonia aetiologies. For example, a large burden of RSV infection in one site will lower the measured vaccine effectiveness even if the two sites have similar vaccine-preventable Hib disease incidences, as has been described previously [26]. Consequently, for clinical (rather than microbiologically confirmed) outcomes, vaccinepreventable disease incidence may be a better measure than vaccine efficacy.

The Indonesian trial by Gessner did not detect an impact on radiological pneumonia [27]. The study authors have proposed several possible explanations why pneumonias with lobar infiltrate or pleural effusion were not preventable with Hib vaccine. First, Hib may not be an important cause of pneumonia in Indonesia; this seems unlikely, since the vaccinepreventable disease incidence measured against all severe or clinical pneumonias was as high or higher than in other studies [24]. Second, some characteristic of children in South East Asia could lead to a different pneumonia presentation; this also seems unlikely since many children presented with a lobar infiltrate meeting the WHO case definition. Last, and perhaps 
most likely, early antibiotic use through selfmedication or early intervention could have modified the evolution of Hib pneumonia [26].

The two remaining studies by Mulholland and Levine found a remarkably similar result of $22 \%$ vaccine efficacy against radiological pneumonia, in spite of different definitions used. In The Gambia, standardized radiology readings were used while in Chile radiology reports were searched for key words, such as 'alveolar consolidation'. The $22 \%$ estimate is, however, less than in three case-control studies from Bangladesh, Dominican Republic and Colombia, which reported effectiveness against radiological pneumonia as $32 \%, 31 \%$ and $55 \%$, respectively [14]. In a study by Theodoratou et al. [28], these three studies were combined with the RCTs of Gessner, Levine and Mulholland and a pooled Hib vaccine effectiveness estimate of $18 \%$ against radiological pneumonia was generated. There was considerable heterogeneity in the pooled estimate, but this problem was not addressed in the study [28].

It must be emphasized that the radiological pneumonia outcome measure imposes a large risk of bias due to the many definition problems of reading chest $\mathrm{X}$-rays. This was particularly illustrated in the casecontrol study from Bangladesh where chest radiographs were taken of 2679 children with clinical pneumonia [29]. According to per-protocol readings, $17 \cdot 7 \%$ cases were identified as having radiologically confirmed pneumonia. However, when the radiographs were read by a WHO panel, it was concluded that $26.0 \%$ were radiologically confirmed, and only $13 \cdot 2 \%$ of cases were radiologically confirmed by both per-protocol readings and WHO readings [29]. It is thus difficult to determine which estimate to use, and the choice greatly affects the vaccine effectiveness estimate. When using the per-protocol readings, the preventable fraction for radiologically confirmed pneumonia was $17 \%$ following at least two doses of Hib vaccine. When the WHO readings were used, the vaccine was associated with $15 \%$ protection, and when the subset considered positive by both sets of readings was used the vaccine offered $34 \%$ protection [29].

The pooled vaccine efficacy estimate against clinical pneumonia appears robust as there is no heterogeneity between the two studies. It is noteworthy that the two studies found such comparable results as there is a high risk of bias with this non-specific outcome measure. However, with only two studies the evidence is limited and as discussed earlier, the estimates cannot be generalized to other settings without caution due to potential fluctuations in clinical pneumonia aetiology between countries and years.

Vaccine efficacy data against non-specific outcomes must be interpreted cautiously. While vaccine efficacy against microbiologically confirmed outcomes reflects the ability of the vaccine to induce protective immunity against infection and disease, vaccine efficacy against non-specific outcomes reflects vaccine performance in combination with the epidemiological context in which the vaccine is used. For example, vaccine efficacy may change by number of doses based on a different distribution of aetiologies at different immunization ages.

The PRP-T and the HbOC vaccines showed similar dose-specific efficacy values, but the PRP-OMP vaccine, which has different kinetics to the other two vaccines, had larger efficacy following one dose. The PRP-OMP vaccine has shown significantly higher immunogenicity after the first dose than the two others in all studies comparing the vaccines [8]. While all three vaccines are indicated for primary infant immunization, PRP-OMP may afford a marginal advantage in populations with high disease burden and carriage prevalence in young infants. For example, in Alaska Native children - who during the pre-vaccine era had among the highest Hib disease incidence rates ever recorded [30]-a change from PRP-OMP to HbOC vaccine in 1996 led to an increase in invasive Hib disease incidence from $19 \cdot 8$ to $91 \cdot 1$ cases/ 100000 children aged $<5$ years [31]. When the state subsequently switched back to PRP-OMP vaccine, disease incidence decreased to $0 / 100000$ in 2004 [31]. Nevertheless, most countries in Africa have reported a high Hib disease burden in young infants prevaccine, and yet have achieved and sustained near elimination of Hib disease with PRP-T [32], indicating this vaccine is also appropriate in these situations.

There was no significant difference between two and three doses, with efficacy against invasive $\mathrm{Hib}$ disease being $92 \%$ and $93 \%$ following two and three doses, respectively. In theory, then, the routine schedule could be reduced to two instead of three doses. A study evaluating this option found that while PRP-T elicited high immune response after two doses, this was not universally true of PRP-HbOC, with only $87 \%$ of infants reaching seroprotective concentrations [33]. The authors concluded that before switching to a two-dose regimen, additional studies would be needed [33]. These studies have not been 
conducted, and moreover, additional barriers to a two-dose schedule exist. First, studies would need to confirm vaccine effectiveness against carriage, since indirect protection is a critical component of overall disease reduction and second, additional studies would need to confirm long-term immunity and protection against carriage, as well as booster responses with a two-dose schedule. Programmatically, Hib vaccine is now widely introduced in combination with DTP and other vaccines with a primary three-dose schedule, which has large advantages. For these reasons, it is unlikely that any country would consider a two-dose primary series without a booster. However, if countries were to switch to a $2+1$ schedule for pneumococcal vaccines, our analysis supports the use of the same schedule for Hib vaccine.

While not evaluated in this study, choices of vaccine formulations and schedules must also consider the immune status of the population, particularly the effects of human immunodeficiency virus (HIV) infection and malnutrition. For example, HIV infection leads to decreased immunogenicity [34]; however, population impact against Hib disease in HIVendemic areas has varied with South Africa showing reduced impact [35] and Malawi showing high impact [36].

Waning immunity and the possible need for a booster dose is another important issue to consider. Since the medium follow-up time of the studies was only 24 months, they do not provide evidence regarding the long-term protection of the vaccine. While all high-income and some middle-income countries recommend a Hib vaccine booster dose at 12-15 months, this has not been included in immunization schedules in low-income countries. The reason for not including a booster dose is partly due to the increased cost, which is not affordable, but also because the average age of Hib infection is substantially lower in low-income compared to high-income countries [37]. It is, however, extremely important to closely monitor the impact of Hib vaccine in lowincome countries to establish whether a booster dose is also needed in these settings. In South Africa an increase in invasive $\mathrm{Hib}$ disease cases was observed several years after a three-dose primary vaccination schedule was commenced. Surveillance data showed that about $60 \%$ of Hib vaccine failures were in HIVnegative children and $55 \%$ occurred in children aged $>18$ months; an age group that may benefit directly from a booster dose. In 2010 South African children began receiving a Hib booster [38].
Boosting is important in sustaining both direct protection and indirect effects through reduction in carriage. Although we could not evaluate the effect of different schedules on carriage, this issue is of primary importance, since much of the overall protection against disease afforded by Hib vaccination programmes derives from indirect effects. In the UK, an increase in vaccine failure 8 years after routine vaccination commenced in 1999 was at least partly because of a greater than expected decline in Hib antibody response following a schedule without a booster in the second year of life, which had been masked by the initial vaccination catch-up programme of all children aged $<5$ years in 1992 [39]. Moreover, the loss of protection against Hib disease in children resulted in a significant increase in adult disease, probably because reduced carriage and transmission in young children had diminished opportunities for natural boosting of immunity in adults, who had very low Hib antibody levels [40]. This suggests that control of carriage and disease in young children has important impact in older children and adults.

Investigators of large-scale vaccination trials do not routinely report dose-specific outcomes despite their potential importance for the impact and costeffectiveness of immunization programmes. Estimates of dose-specific vaccine efficacy provide a direct way for analysts and decision makers to account for vaccine impact in partially vaccinated children. Models that do not account for partial protection will underestimate overall programme effectiveness in populations with high drop-out rates between doses.

\section{ACKNOWLEDGEMENTS}

We thank Dr Phil Edwards for useful discussions on meta-analysis methods and an anonymous reviewer for important suggestions.

This paper is dedicated to the memory of our co-author, colleague, and friend, Dr Endang Sedyaningsih, the Indonesian Minister of Health, who died on 2 May 2012.

\section{DECLARATION OF INTEREST}

None.

\section{REFERENCES}

1. Peltola H. Worldwide Haemophilus influenzae type b disease at the beginning of the 21st century: global analysis of the disease burden 25 years after the use of 
the polysaccharide vaccine and a decade after the advent of conjugates. Clinical Microbiology Reviews 2000; 13: 302-317.

2. WHO. Vaccine-preventable disease monitoring system. Global and regional immunization profile (http:// wwwwhoint/immunization_monitoring/en/globalsum mary/GS_GLOProfilepdf). Accessed December 2011.

3. Zuber PL, et al. Sustaining GAVI-supported vaccine introductions in resource-poor countries. Vaccine 2011; 29: 3149-3154.

4. Kelly DF, Moxon ER, Pollard AJ. Haemophilus influenzae type b conjugate vaccines. Immunology 2004; 113: 163-174.

5. Eskola J, et al. A randomized, prospective field trial of a conjugate vaccine in the protection of infants and young children against invasive Haemophilus influenzae type b disease. New England Journal of Medicine 1990; 323: 1381-1387.

6. Ward J, et al. Limited efficacy of a Haemophilus influenzae type $b$ conjugate vaccine in Alaska Native infants. The Alaska H. influenzae Vaccine Study Group. New England Journal of Medicine 1990; 323: 13931401.

7. WHO. WHO Position Paper on Haemophilus influenzae type b conjugate vaccines Weekly Epidemiological Record 2006; 81: 445-452.

8. Capeding MR, et al. The immunogenicity of three Haemophilus influenzae type B conjugate vaccines after a primary vaccination series in Philippine infants. American Journal of Tropical Medicine and Hygiene 1996; 55 : 516-520.25.

9. Clemens J, et al. Evaluating new vaccines for developing countries. Efficacy or effectiveness? Journal of American Medical Association 1996; 275: 390-397.

10. Griffiths UK, Miners A. Economic evaluations of Haemophilus influenzae type b vaccine: systematic review of the literature. Expert Review of Pharmacoecononics and Outcomes Research 2009; 9: 333-346.

11. Clark A, Sanderson C. Timing of children's vaccinations in 45 low-income and middle income countries: an analysis of survey data. Lancet 2009; 373: 1543-1549.

12. Fitzwater SP, et al. Haemophilus influenzae type b conjugate vaccines: considerations for vaccination schedules and implications for developing countries. Human Vaccines 2010; 6: 810-818.

13. Obonyo CO, Lau J. Efficacy of Haemophilus influenzae type b vaccination of children: a meta-analysis. European Journal of Clinical Microbiology and Infectious Diseases 2006; 25: 90-97.

14. O'Loughlin RE, et al. Methodology and measurement of the effectiveness of Haemophilus influenzae type $b$ vaccine: systematic review. Vaccine 2010; 28: 61286136.

15. Liberati A, et al. The PRISMA statement for reporting systematic reviews and meta-analyses of studies that evaluate health care interventions: explanation and elaboration. PLoS Medicine 2009; 6: e1000100.

16. Dickersin K, et al. Development of the Cochrane Collaboration's CENTRAL Register of controlled clinical trials. Evaluation \& the Health Professions 2002; 25: $38-64$.

17. Higgins JPT, Green, S (eds). Cochrane Handbook for Systematic Reviews of Interventions. The Cochrane Collaboration, March 2011.

18. Cherian T. Describing the epidemiology and aetiology of bacterial pneumonia in children: an unresolved problem. Journal of Health Population and Nutrition 2005; 23 : 1-5.

19. Shann F. Determining etiology of pneumonia. Pediatric Infectious Disease Journal 1995; 14: 920-921.

20. Obaro SK, Madhi SA. Bacterial pneumonia vaccines and childhood pneumonia: are we winning, refining, or redefining? Lancet Infectious Diseases 2006; 6: 150-161.

21. Mulholland K, et al. Randomised trial of Haemophilus influenzae type-b tetanus protein conjugate for prevention of pneumonia and meningitis in Gambian infants. Lancet 1997; 349: 1191-1197.

22. Deeks JJ, Altman, DG, Bradburn, MJ. Statistical methods for examining heterogeneity and combining results from several studies in meta-analysis. In: Egger, M, Smith, GD, Altman, DG, eds. Systematic Reviews in Health Care Meta-analysis in Context: BMJ Books, 2001.

23. Higgins JP, et al. Measuring inconsistency in metaanalyses. British Medical Journal 2003; 327: 557-560.

24. Peltola H, et al. Clinical comparison of the Haemophilus influenzae type B polysaccharide diphtheria toxoid and the oligosaccharide-CRM197 protein vaccines in infancy. Archives of Pediatrics \& Adolescent Medicine 1994; 148: 620-625.

25. Santosham M, et al. The efficacy in Navajo infants of a conjugate vaccine consisting of Haemophilus influenzae type b polysaccharide and Neisseria meningitidis outermembrane protein complex. New England Journal of Medicine 1991; 25: 1767-1772.

26. Gessner BD. Haemophilus influenzae type b vaccine impact in resource-poor settings in Asia and Africa. Expert Review Vaccines 2009; 8: 91-102.

27. Gessner BD, et al. Incidences of vaccine-preventable Haemophilus influenzae type b pneumonia and meningitis in Indonesian children: hamlet-randomised vaccine-probe trial. Lancet 2005; 365: 43-52.

28. Theodoratou E, et al. The effect of Haemophilus influenzae type $\mathrm{b}$ and pneumococcal conjugate vaccines on childhood pneumonia incidence, severe morbidity and mortality. International Journal of Epidemiology 2010; 39 (Suppl. 1): i172-185.

29. Baqui AH, et al. Effectiveness of Haemophilus influenzae type $b$ conjugate vaccine on prevention of pneumonia and meningitis in Bangladeshi children: a case-control study. Paediatric Infectious Disease Journal 2007; 26: 565-571.

30. Ward JI, et al. Invasive Haemophilus influenzae type b disease in Alaska: background epidemiology for a vaccine efficacy trial. Journal of Infectious Diseases 1986; 153: 17-26.

31. Singleton R, et al. The Alaska Haemophilus influenzae type $\mathrm{b}$ experience: lessons in controlling a vaccinepreventable disease. Pediatrics 2006; 118: e421-429. 
32. Cowgill KD, et al. Effectiveness of Haemophilus influenzae type b conjugate vaccine introduction into routine childhood immunization in Kenya. Journal of American Medical Association 2006; 296: 671-678.

33. Lagos R, et al. Economisation of vaccination against Haemophilus influenzae type $\mathrm{b}$ : a randomised trial of immunogenicity of fractional-dose and two-dose regimens. Lancet 1998; 351 : 1472-1476.

34. Mangtani P, et al. Haemophilus influenzae type b disease in HIV-infected children: a review of the disease epidemiology and effectiveness of Hib conjugate vaccines. Vaccine 2010; 28: 1677-1683.

35. Madhi SA, et al. Reduced effectiveness of Haemophilus influenzae type $b$ conjugate vaccine in children with a high prevalence of human immunodeficiency virus type 1 infection. Pediatric Infectious Disease Journal 2002; 21: 315-321.

36. Daza P, et al. The impact of routine infant immunization with Haemophilus influenzae type $\mathrm{b}$ conjugate vaccine in Malawi, a country with high human immunodeficiency virus prevalence. Vaccine 2006; 24: 6232-6239.

37. Bennett JV, et al. Haemophilus influenzae type b (Hib) meningitis in the pre-vaccine era: a global review of incidence, age distributions, and case-fatality rates. WHO/V\&B/0218. Geneva: WHO, 2002.

38. von Gottberg A CC, et al. Invasive disease due to Haemophilus influenzae serotype b ten years after routine vaccination, South Africa, 2003-2009. Vaccine 2012; 30: 565-71.
39. Ramsay ME, et al. Estimating Haemophilus influenzae type $b$ vaccine effectiveness in England and Wales by use of the screening method. Journal of Infectious Diseases 2003; 188: 481-485.

40. McVernon J, et al. Trends in Haemophilus influenzae type $\mathrm{b}$ infections in adults in England and Wales: surveillance study. British Medical Journal 2004; 329: 655-658.

41. Black SB, et al. Efficacy in infancy of oligosaccharide conjugate Haemophilus influenza type b (HbOC) vaccine in a United States population of 61,080 children. The Northern California Kaiser Permanente Vaccine Study Center Pediatrics Group. Pediatric Infectious Disease Journal 1991; 10: 97-104.

42. Booy R, et al. Efficacy of Haemophilus influenzae type b conjugate vaccine PRP-T. Lancet 1994; 344: 362-366.

43. Lagos R, et al. Large scale, postlicensure, selective vaccination of Chilean infants with PRPT conjugate vaccine: Practicality and effectiveness in preventing invasive Haemophilus influenzae type $\mathrm{b}$ infections. Pediatric Infectious Disease Journal 1996; 15: 216-222.

44. Levine OS, et al. Defining the burden of pneumonia in children preventable by vaccination against Haemophilus influenzae type b. Pediatric Infectious Disease Journal 1999; 18: 1060-1064.

45. Vadheim CM, et al. Effectiveness and safety of a Haemophilus influenzae type b conjugate vaccine (PRP$\mathrm{T})$ in young infants. Kaiser-UCLA Vaccine Study Group. Pediatrics 1993; 92: 272-279. 